\title{
Coupling of plasmonic nanopore pairs: facing dipoles attract each other
}

\author{
Takumi Sannomiya $^{1}$, Hikaru Saito ${ }^{2}$, Juliane Junesch ${ }^{3}$ and Naoki Yamamoto ${ }^{1}$
}

Control of the optical properties of nano-plasmonic structures is essential for next-generation optical circuits and high-throughput biosensing platforms. Realization of such nano-optical devices requires optical couplings of various nanostructured elements and field confinement at the nanoscale. In particular, symmetric coupling modes, also referred to as dark modes, have recently received considerable attention because these modes can confine light energy to small spaces. Although the coupling behavior of plasmonic nanoparticles has been relatively well studied, couplings of inverse structures, that is, holes and pores, remain partially unexplored. Even for the most fundamental coupling system of two dipolar holes, comparison of the symmetric and antisymmetric coupling modes has not been performed. Here we present, for the first time, a systematic study of the symmetric and anti-symmetric coupling of nanopore pairs using cathodoluminescence by scanning transmission electron microscopy and electromagnetic simulation. The symmetric coupling mode, approximated as a pair of facing dipoles, is observed at a lower energy than that of the anti-symmetric coupling mode, indicating that the facing dipoles attract each other. The anti-symmetric coupling mode splits into the inner- and outer-edge localized modes as the coupling distance decreases. These coupling behaviors cannot be fully explained as inverses of coupled disks. Symmetric and anti-symmetric coupling modes are also observed in a short-range ordered pore array, where one pore supports multiple local resonance modes, depending on the distance to the neighboring pore. Accessibility to the observed symmetric modes by far field is also discussed, which is important for nanophotonic device applications.

Light: Science \& Applications (2016) 5, e16146; doi:10.1038/lsa.2016.146; published online 9 Septemeber 2016

Keywords: cathodoluminescence; plasmonic nanopore; short-range order; surface plasmon; transmission electron microscopy

\section{INTRODUCTION}

Nano-optical devices are expected to become the next generation of computing circuits, high-throughput optical biosensors and energy harvesters. Especially, plasmonic nanostructures are promising elements because they can be down-sized below the wavelength. In real devices, each plasmonic element from the input to the output should be optically coupled to perform a certain function. For several years, symmetric coupling modes (also referred to as 'dark modes') with symmetric charge distribution, which in principle cannot be excited by plane-wave illumination along the symmetry axis, have been drawing attention because they can support more confined fields and have higher Q-factors than the anti-symmetric (so-called 'bright') modes. Possible applications include high-sensitivity sensors, lasing, light emission enhancement and miniaturized plasmonic waveguides ${ }^{1-6}$. To utilize these advantages, coupled nanoparticle systems, including nanodisks and nanorods, have been intensively studied ${ }^{1-6}$. Previous studies have demonstrated that the symmetric mode of plasmonic particle pairs has a higher energy than the anti-symmetric mode and that the coupling energy increases as the inter-particle distance decreases, which can be explained by repulsive dipoles ${ }^{1,2,5,6}$. In contrast, investigations of the coupling of nanoholes and nanopores have been limited to the anti-symmetric coupling modes in the array configuration, and symmetric modes have not been discussed, although various promising applications using nanopores and nanoholes in sensing and optoelectronics have been proposed ${ }^{1-4,7-11}$. Whereas anti-symmetric modes ('bright modes') of arrays, chains and isolated single holes are relatively well studied, pairs have not been studied, although they are the most fundamental and simplest coupling models ${ }^{7-13}$. The limitation for investigating symmetric modes is related to the measurement method; simple, far-field instrumentation cannot selectively resolve these modes because the symmetry of the excited mode is limited by the symmetry of the plane wave with far-field excitation. In this study, we investigate, for the first time, the symmetric and anti-symmetric coupling of plasmonic nanopore pairs and reveal the unique coupling behavior of hole/pore systems. We employed cathodoluminescence (CL) and electron energy loss spectroscopy (EELS) using a Cs-corrected scanning transmission electron microscope (STEM), where a focused electron beam of $1 \mathrm{~nm}$ in diameter serves as a point source to selectively excite the modes according to the symmetry and beam position ${ }^{14-18}$. In CL imaging, the radiative electromagnetic local density of state (EMLDOS) can be mapped, which corresponds to the electric field distribution along the

${ }^{1}$ Department of Innovative and Engineered Materials, Tokyo Institute of Technology, Yokohama 226-8503, Japan; ${ }^{2}$ Center of Advanced Instrumental Analysis, Kyushu University, Kasuga 816-8580, Japan and ${ }^{3}$ Department of Physics, Chalmers University of Technology, Gothenburg 41296, Sweden

Correspondence: T Sannomiya, Email: sannomiya.t.aa@m.titech.ac.jp

Received 28 September 2015; revised 7 March 2016; accepted 5 April 2016; accepted article preview online 7 April 2016 
electron path ${ }^{19-21}$. The far-field light intensity is detected in CL measurement, indicating that the detected modes are actually accessible by far field on the basis of optical reciprocity, whereas detection by EELS in STEM is not directly related to far-field light interaction. Because so-called 'dark modes' can be detected as radiated light at high angles in CL measurement, we use the term 'symmetric' rather than 'dark mode' in this study.

\section{MATERIALS AND METHODS}

\section{Cathodoluminescence}

In our CL-STEM system, the radiated light is collimated by a parabolic mirror placed around the specimen and is detected by a spectrometer (Figure 1a). With a large solid angle of light collection by the parabolic mirror around the specimen (solid angle: $3 \pi$ ), except for the shading by the sample holder, symmetric modes can be detected ${ }^{14,15,22}$. In this study, the polarization is perpendicular to the electron and light optical axes, as shown in Figure 1a. This polarization setting filters out a large portion of the field component along the axis of the optical detection, probably due to the combination of beaming from nanoholes and angle-dependent dipolar radiation ${ }^{13,23}$. A JEM-2100F (JEOL, Tokyo, Japan) transmission electron microscope with a Schottky field emission electron source was used, and the acceleration voltage of the electron beam was $80 \mathrm{kV}$. The equipped Cs-corrected system allows a large convergence angle of the electron beam of $\sim 20 \mathrm{mrad}$ and a beam current of $5 \mathrm{nA}$, with $\sim 1$-nm beam diameter. a

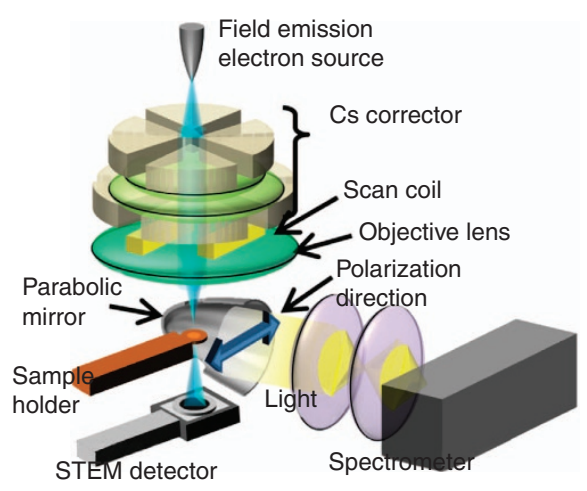

b

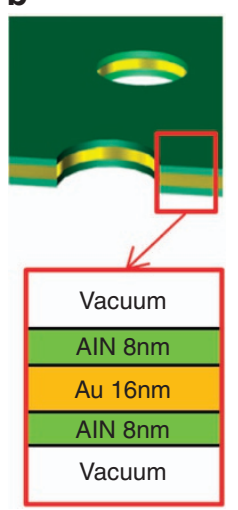

C

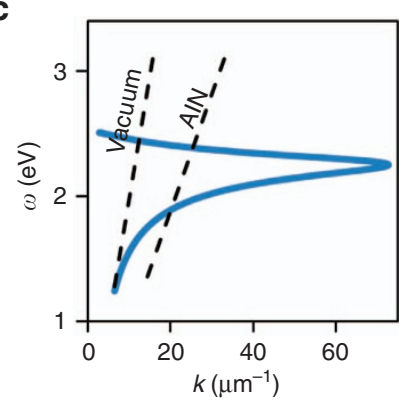

d

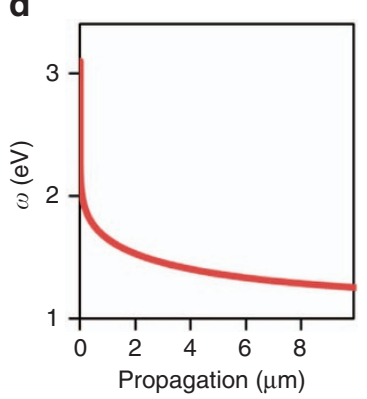

Figure 1 Illustration of CL-STEM measurement and nanopore charcteristics. (a) Schematic illustration of the CL-STEM setup. The polarization direction is shown by the arrow. (b) Illustration of the nanopore specimen with the diameter of $135 \mathrm{~nm}$. (c) Dispersion relation of the bonding (short-range) SP mode of the $\operatorname{AIN}(8 \mathrm{~nm}) / \mathrm{Au}(16 \mathrm{~nm}) / \operatorname{AIN}(8 \mathrm{~nm})$ trilayer structure. The light lines are presented as dotted lines. (d) Corresponding propagation length of the SP.

\section{Nanopore fabrication}

Nanopore samples were prepared as previously described ${ }^{24}$. In short, nanopores were fabricated by colloidal lithography and film transfer by wet-etching of the sacrificial layer. To achieve very close separation of nanopore pairs and to obtain high spatial resolution in STEM, we chose ultra-thin, free-standing film structures. For the measurement of single and coupled pairs of nanopores, $135 \mathrm{~nm}$ nanopores in an $\operatorname{AlN}(8 \mathrm{~nm}) / \mathrm{Au}(16 \mathrm{~nm}) / \mathrm{AlN}(8 \mathrm{~nm})$ trilayer membrane were used (Figure 1b). With this sandwich layer structure, it is possible to obtain very thin and stable metal layers, even at high temperatures ${ }^{25,26}$. To achieve separated structures with small gaps for pairs, the carbonmediated method was applied ${ }^{24}$. For the investigation of short-range ordered (SRO) nanopores, $\operatorname{AlN}(10 \mathrm{~nm}) / \mathrm{Au}(12 \mathrm{~nm}) / \mathrm{AlN}(6 \mathrm{~nm})$ trilayer films with $120 \mathrm{~nm}$ nanopores were fabricated using the directetching method, which is suited for dense pore packing ${ }^{24}$. The AlN layers work both as a buffer layer and to stabilize the gold film. The dimensions were chosen so that the resonance is in the visible-nearinfrared wavelength. To tune the resonance in the measurable wavelength range and to investigate the coupling of nanopores through surface plasmons (SPs), the dispersion relation and the propagation length of the bonding (or short-range) mode SP, which is predominantly excited by a fast electron beam, were calculated

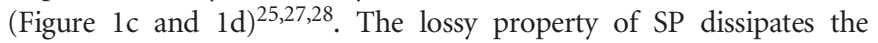
nanohole resonances and reduces the Q-factor and field confinement. Due to this dissipation, the high mode density of $\sim 2.2 \mathrm{eV}$ does not directly contribute to the CL signal.

\section{Simulation}

Simulation was performed to analyze the resonance modes found in the experimental measurement and their behavior. Because the CL and EELS signals correspond to the radiative and full EMLDOS along the electron path ( $z$ axis), the calculated field intensity in the $z$ direction with far-field excitation roughly represents the CL signal based on the reciprocity. The electromagnetic field calculation was conducted by the finite-difference time-domain (FDTD) method using the CrystalWave software package (Photon Design, Oxford, UK). FDTD can identify the dominant resonance mode by a pulse excitation wave and can avoid undesired SP reflection from the truncation edges by analyzing the field after the pulse has passed but before the reflection reappears (more details can be found in the Supplementary Information). This reflection becomes non-negligible for isolated structures in metallic films, which gives inaccurate results in frequency domain methods. Because the radiated light is detected in the CL measurement, we excited all modes, including the symmetric modes, by plane-wave illumination and detected the perpendicular field close to the sample as the reciprocal of the CL measurement. The anti-symmetric mode is excited by a plane wave with a normal incidence to the film plane with the electric field polarized along the coupling axis. Two detectors were set at the inner and outer edges to detect the inner- and outer-edge modes. The symmetric mode is excited by two plane waves tilted by $30^{\circ}$ from normal to the coupling axis directions. Both plane waves are polarized along the coupling axis and illuminated with the same phase to maintain the symmetry, and the perpendicular electric field is detected at the center.

\section{RESULTS AND DISCUSSION}

\section{Coupled nanopore pairs}

As an initial investigation of single and coupled pairs of nanopores, $135 \mathrm{~nm}$ nanopores in an $\operatorname{AlN}(8 \mathrm{~nm}) / \mathrm{Au}(16 \mathrm{~nm}) / \mathrm{AlN}(8 \mathrm{~nm})$ trilayer membrane were measured (Figure $1 \mathrm{~b}$ ). Figure 2 presents the STEM bright-field images, panchromatic photon maps (non-filtered) and 


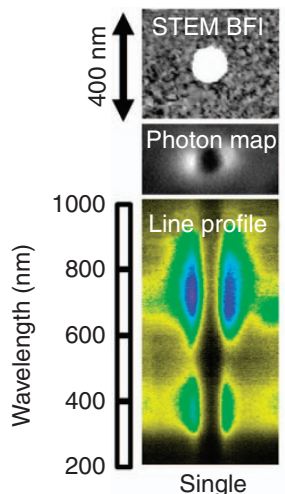

Single

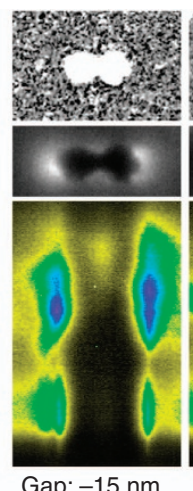

Gap: -15 nm

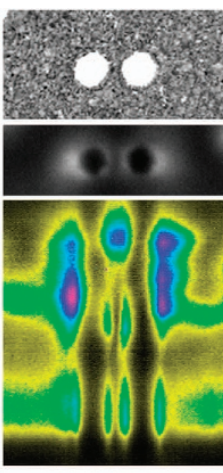

$47 \mathrm{~nm}$

Min
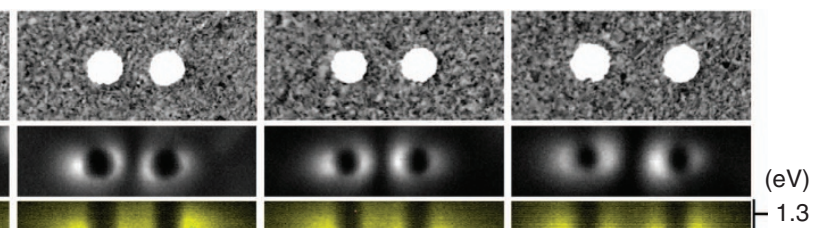

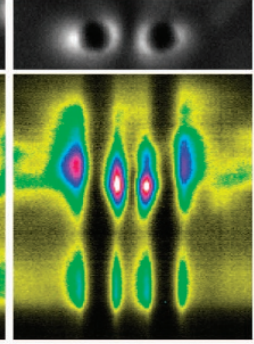

$93 \mathrm{~nm}$

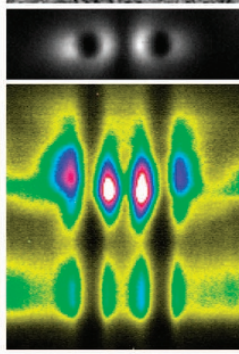

$126 \mathrm{~nm}$
(eV)

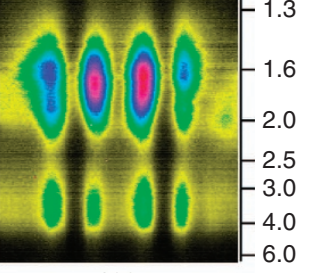

$193 \mathrm{~nm}$

Figure 2 STEM bright-field images (BFIs), photon maps (panchromatic) and horizontal CL spectral line profiles at the nanopore center of single and coupled plasmonic nanopores. The photon map includes photons of all detectable energies (300-1000 nm). The spectral line profile is plotted along the center of the pores (horizontal direction in the image). The detected light is polarized horizontally in the images.

spectral line profiles of the measured nanopore pairs with different 'gap' distances as well as those of a single nanopore. The panchromatic images show that the polarization setup, shown in Figure 1a, mostly extracts the polarization perpendicular to the optical detection axis and electron path (the same polarization is also on the specimen in Figure 1a and the horizontal axis in Figure 2). The details of the polarization dependence of the nanoholes are currently under investigation. The spectral line profiles shown in the lower row in Figure 2 are measured along the center of the pores. The extracted one-dimensional spectra are found in the Supplementary Information. The constant energy band near the wavelength of $\sim 400 \mathrm{~nm}$ is attributed to the inter-band transition of gold. The CL spectra of nanopore pairs with different gap distances reveal that the wavelength of the resonance excited at the inner edge of the gap (the inner-edge mode) exhibits a positive correlation with the gap distance, whereas this tendency of the outer-edge mode, excited at the outer edge, is less steep, although showing a similar gap dependence (Figure 3 ). The inner-edge mode is regarded as an anti-symmetric coupling mode or a so-called bright coupling mode because this mode cannot be excited by the beam placed at the center ${ }^{8,9,15}$. In comparison with the SP wavelength (Figure 1c), the nanopores are located too close to each other, and the pores are considered to be optically coupled through the near field (Supplementary Information). Similar to the inner-edge mode, the outer-edge mode can also be excited by anti-symmetric beam position, which should also be an anti-symmetric mode.

In addition to these anti-symmetric modes appearing at the edge of the pores, a symmetric coupling mode is excited when the electron beam is incident at the center of the gap ${ }^{6,29}$. The symmetric mode can also be weakly excited by illuminating the edges when the gap is small. This symmetric mode always appears at a longer wavelength than the anti-symmetric coupling modes (Figure 3 ). The resonance wavelength of the symmetric mode shows a slight inverse dependence on the gap distance and is more strongly excited when the gap distance is smaller. The symmetric mode still exists even when the gap distance is negative (thus, when the pores are connected). In contrast, the inner-edge, anti-symmetric mode becomes weaker as the gap distance decreases and completely disappears when the pores are connected.

We also performed EELS measurements, which supported the modes found in the CL measurement (Supplementary Information). The EELS (full EMLDOS) measurement confirms that the modes found in the CL (radiative EMLDOS) measurement are not

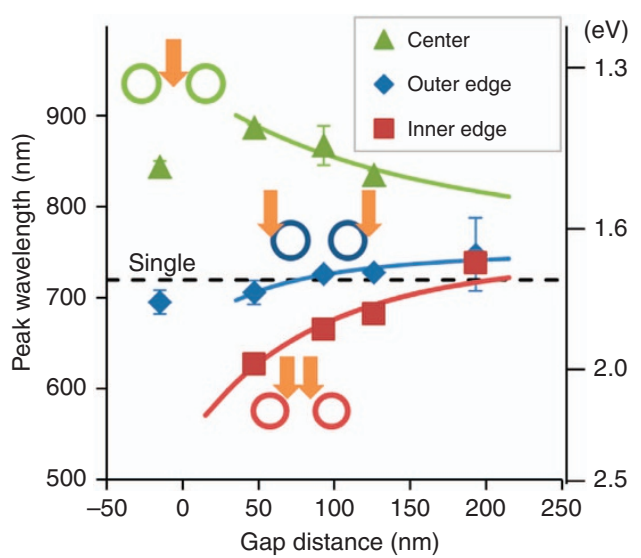

Figure 3 Resonance wavelengths plotted as a function of the 'gap' distance. The fitted solid lines are drawn to guide the eye. The resonance of the single pore is indicated by the dashed line.

significantly influenced by the interference in radiation ${ }^{21}$. To further confirm the inner- and outer-edge modes, we examined a more mode-separated nanopore pair with larger pore sizes, where CL mapping for the mode separation can be more clearly performed than with a system with inner- and outer-edge modes at close energies. We observed the outer-edge mode with low CL intensity distribution at the gap at a longer wavelength, and the inner-edge mode with highly localized field in the gap at a shorter wavelength (Supplementary Information). These complementary measurements confirm the presence and relation of the symmetric mode as well as anti-symmetric inner- and outer-edge modes.

To elucidate and analyze the experimental results, we performed FDTD simulations, as presented in Figures 4 and 5. We first simulated a single pore, as shown in Figure 4. The resonance wavelength of the single pore roughly matches the experimental result. The deviation could be due to the difference of the dielectric constant of the metal and dimensional errors, including the edge shapes and thickness ${ }^{30}$. The electric field patterns $(E z, E x)$ confirm that the SP bonding mode (or short-range SP mode) is dominant. Inside the pore, the electric field is polarized in the $x$ direction, showing a typical $x$-polarized electric dipole. The magnetic field curls around this electric dipole at 
a

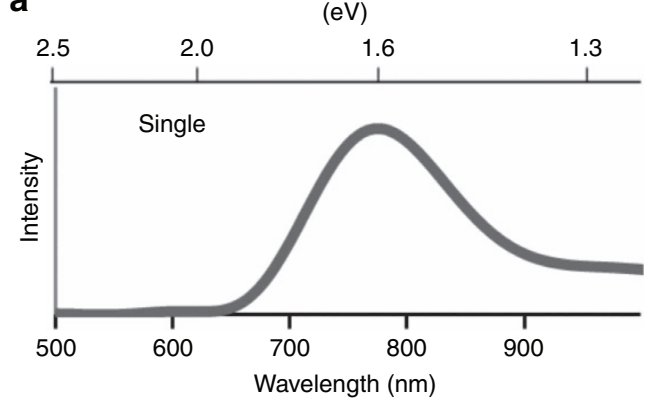

b

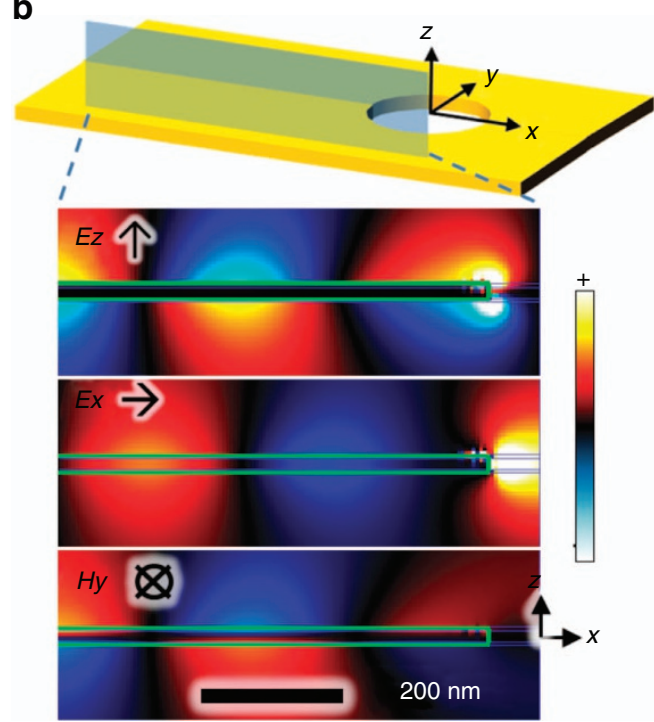

Figure 4 Electromagnetic simulation of a single nanopore. Simulated (a) spectrum and (b) cross-sectional field maps of a single plasmonic nanopore. The pore is excited by a plane wave with normal incidence, and the field intensity at the edge was detected. For the field pattern, components of the electric $(\vec{E})$ and magnetic $(\vec{H})$ fields are plotted at the resonance wavelength. The schematic illustration shows the position of the coordination and the extracted cross-sectional area. The film structure is indicated by green lines in the field maps.

the pore position. Far-field patterns and comparison with a simple electric dipole are shown in the Supplementary Information.

In Figure 5, the simulation results of coupled pores are shown. The anti-symmetric mode spectra detected at the inner-edge and outeredge positions (blue and red lines in Figure 5a) show resonance peaks at a wavelength close to that of the single mode at the gap distance of $200 \mathrm{~nm}$. These two anti-symmetric modes start to blueshift, and the difference of the peak wavelengths increases as the gap distance decreases. The inner-edge mode blueshifts more than the outer-edge mode. The symmetric mode starts to appear and becomes stronger as the gap distance decreases, which is opposite to the anti-symmetric modes. The symmetric mode resonance always appears at longer wavelengths than that of the anti-symmetric or single modes. The simulated in-plane field pattern of the symmetric mode is similar to the wave propagation of the breathing mode $\mathrm{e}^{31}$. Although the simulation of the spectra qualitatively reproduces the experimental results, the quantitative differences originate from slight differences in dimensions, fabrication errors or differences in the material constants of gold and aluminum nitride ${ }^{32}$. In addition, the intensity analysis also shows agreement of the experiment and simulation (Supplementary Information). The simulation results show that the symmetric modes can be excited by oblique plane-wave incidence, although we took advantage of the symmetric illumination by two in-phase plane waves to avoid excitation of the anti-symmetric modes. This result also indicates that symmetric modes can be detected by far-field optical measurement with symmetric excitation and detection. However, due to coupling with the propagating waves, the observed symmetric mode does not necessarily have sharper peaks than the anti-symmetric mode (low Q-factors), which also makes the determination of the resonance erroneous.

To better understand the two different anti-symmetric modes, namely, the inner- and outer-edge modes, the perpendicular electric field $(E z)$ patterns are plotted in Figure 5b. From the reciprocity of the CL signal, $E z$ corresponds to the CL signal ${ }^{15,19-21}$. The three images with solid line frames show the electric field patterns of the bright inner-edge mode, bright outer-edge mode and symmetric mode at the peak wavelengths indicated by the arrows in Figure $5 \mathrm{a}$. The field intensity of the inner-edge mode is strongly localized at the inner edge, and each pore behaves like a dipole. In contrast, the field of the outeredge mode is propagating outside the pore and has very low intensity at the gap position. Judging from the field pattern close to the pore, this mode may be considered as coupling of the quadrupoles formed around each pore. We found another weak, outer-edge, antisymmetric mode from dipoles at a much longer wavelength in the simulated spectrum (dashed blue arrow in Figure $5 \mathrm{a}$ and frame in Figure $5 b$, also in the Supplementary Information).

Because the inner-edge mode is strongly localized at the gap and the outer-edge mode has almost no field at the gap for small gap distances, we assumed that the local structure determines the resonance mode rather than the whole structure of the nanopore pair. To extract only the features of the gap, we modeled a structure without outer edges, consisting of two semi-infinitely long slits (details in the Supplementary Information). The simulated spectra of this 'only-gap structure' match the spectral responses of the inner-edge mode, and the field patterns resemble each other. This result indicates that the gap structure only determines the inner-edge mode, with minimal influence of the outer edge. On the basis of the idea of this mode localization, the inner-edge mode can be interpreted as a thin metal strip. Then, the gap distance dependence of the energy of the inneredge mode can be understood from charge distributions of thin-film plasmons ${ }^{33,34}$. As an extreme case of the outer-edge mode, we modeled a single slit with the same width as the outer edge-to-edge distance (Supplementary Information). This structure reproduced similar resonances and field pattern as the outer-edge mode.

For intuitive understanding and simpler analysis, hole or pore structures are often treated as inverse particles because particles are better understood as interacting dipole charges ${ }^{35,36}$. For the best simplicity, a thin metal film is considered as an infinitely thin perfect electric conductor (PEC). With this thin-film approximation and by applying Babinet's principle, the symmetric coupling of pores corresponds to the anti-parallel coupling of disk particles with a side-to-side

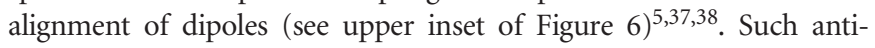
parallel configuration has lower energy than the parallel coupling configuration. With this approximation, the relation between the symmetric and anti-symmetric modes can be intuitively understood. However, this exchange of the electric and magnetic fields using Babinet's principle does not simply convert the system to electric dipole disks, according to the field patterns at the pore, as shown in Figure 4 (the magnetic field is curling around the electric dipole). The coupled disks regarded as inverse structures cannot explain the separate inner- and outer-edge coupling modes, as summarized in Figure 6. We note that the coupling energies found in this study do 
not fully agree with the previously proposed models for the chain configuration, where not all of the modes were resolved and analyzed due to complexity ${ }^{39}$. For such coupled plasmonic nanopores in a thin membrane, inversing the pore structures to disks does not completely

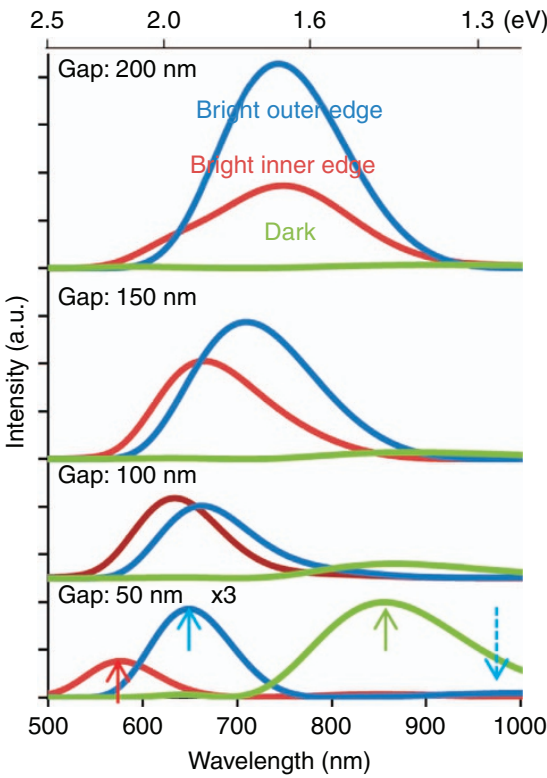

b

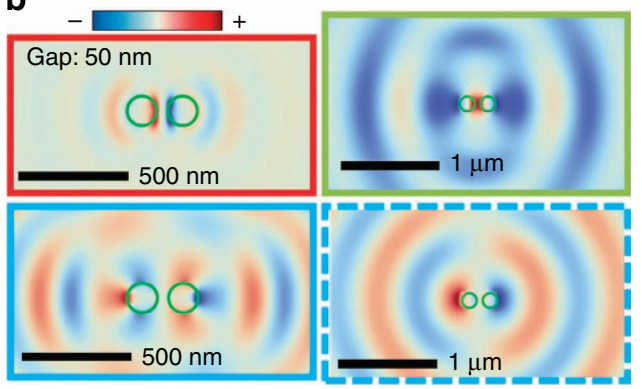

Figure 5 Simulation of the coupled plasmonic nanopores. The antisymmetric modes are excited by a plane wave with normal incidence, and the symmetric mode is excited by two plane waves with $\pm 30^{\circ}$ incident angles. The field intensity was detected from the other side of the membrane at the corresponding positions. (a) The spectra of the antisymmetric and symmetric coupling modes. The intensities for the 50-nm gap spectra are tripled for visibility. (b) The in-plane pattern of the perpendicular electric field (Ez) for a 50-nm gap distance at the wavelengths indicated by arrows in a. The color and style of the arrows correspond to those of the frame of the field pattern. explain the coupling behavior, although it partially facilitates the interpretation. To fully understand the plasmonic structure with propagating SPs, it is important to consider the local modes. Therefore, nanoscopic optical observation is essential.

\section{Local coupling in SRO arrays}

Because the resonance modes can be localized when the pores are close to each other, we investigated a system consisting of multiple pores with various distances, which comprises a SRO nanopore array ${ }^{37}$. We used an $\operatorname{AlN}(10 \mathrm{~nm}) / \mathrm{Au}(12 \mathrm{~nm}) / \operatorname{AlN}(6 \mathrm{~nm})$ trilayer with a pore diameter of $120 \mathrm{~nm}$ (Figure 7a). The line profiles across adjacent nanopores in Figure 7a exhibit both symmetric and anti-symmetric modes, similar to the nanopore pairs discussed above (Figure 2). One pore shows different resonance wavelengths at the left and right edges when the distances between the left and right neighbors are different. This difference resembles the strong localization of the inner-edge mode, which is insensitive to the outside environment, as discussed previously for pairs. The resonance wavelengths plotted in Figure $7 \mathrm{~b}$ exhibit the same trend observed for the isolated nanopore pairs shown in Figure 3: the symmetric mode (the gap center excitation) is located at a lower energy than the anti-symmetric mode (the inner-edge excitation), and its resonance wavelength demonstrates a slight inverse dependence on the gap distance, whereas that of the anti-symmetric mode becomes strongly redshifted as the gap distance increases. The shift of the resonance wavelengths with the gap distance is rapid compared with that observed for the isolated pairs (Figure 3), which can be attributed to the shorter SP wavelength in this layer system (Supplementary Information). These two modes correspond to the $\omega+$ and $\omega-$ modes with different node positions in a long-range periodic array, which cause the energy gap in the photonic band structure $^{40}$

\section{CONCLUSIONS}

We have shown the unique coupling behavior of coupled plasmonic nanopores using Cs-corrected CL-STEM. The symmetric coupling mode exhibits a lower-energy resonance than the anti-symmetric mode, and the resonance wavelength of the anti-symmetric mode exhibits a strong positive correlation with the gap distance. The inneredge, anti-symmetric coupling mode is strongly localized at the gap, which explains the local resonance modes in SRO plasmonic pores. In contrast, the outer-edge mode has almost no field at the gap. Babinet's principle, assuming inverse structures in thin PEC films, does not fully explain such coupling of plasmonic nanopores, although the qualitative comparison of the symmetric and anti-symmetric modes is still valid. Such understanding of field localization of symmetric and antisymmetric modes and the relation of the coupling energy provides an important foundation for designing structures to confine light field at the nanoscale ${ }^{1-4}$. Furthermore, it is shown that a nanoscopic
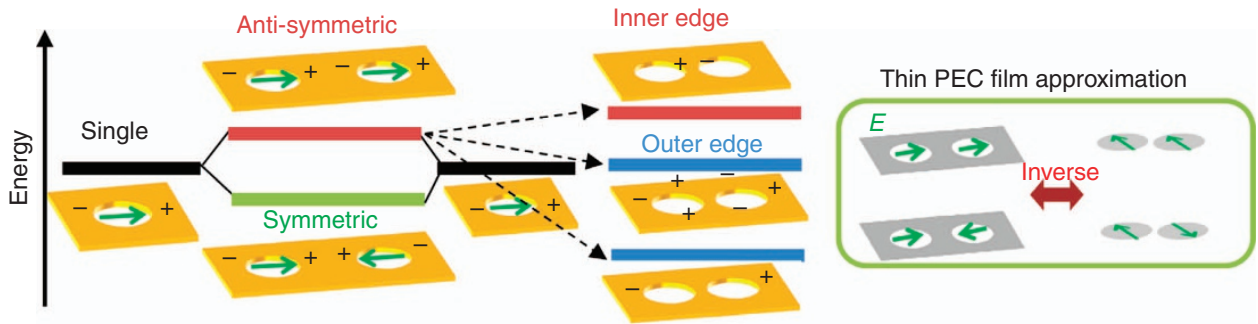

Figure 6 Schematic illustration of the single dipole mode, anti-symmetric dipole coupling and symmetric dipole coupling of plasmonic nanopores. Illustration of the thin PEC film approximation with inverse structures is also shown. 
a

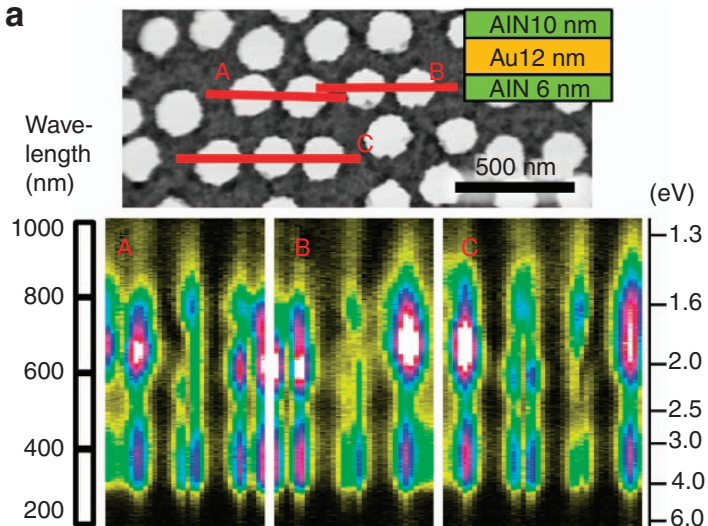

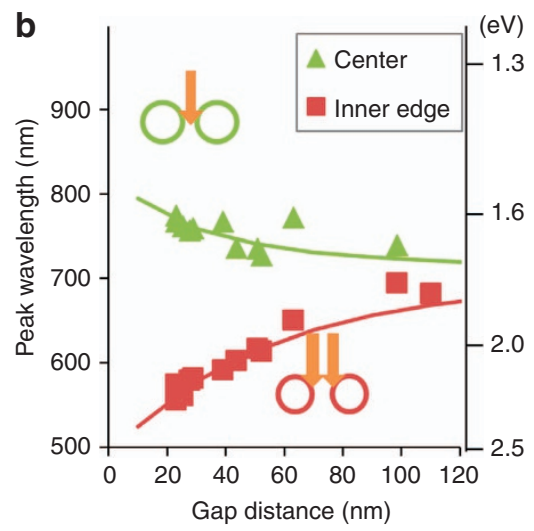

Figure 7 CL-STEM measurement of SRO nanopores. (a) STEM bright-field image (BFI) of the SRO plasmonic nanopores and the CL line profiles from the three locations shown in the BFI. Illustration of the layer structure is shown in the inset. The pore diameter is $120 \mathrm{~nm}$. (b) Resonance wavelength plot of the symmetric and anti-symmetric modes with various local gap distances. Both symmetric (center) and anti-symmetric (inner edge) modes are observed when the gap is small enough. The fitted lines are drawn to guide the eyes.

observation, such as STEM-CL measurement, is essential to analyze field localization and resonance modes. The insights of nanopore couplings obtained here would be useful for sensor applications taking advantage of pore shapes as well as for sub-wavelength photonic devices ${ }^{41,42}$. We have also shown that the observed coupling modes are accessible by obliquely incident plane waves, which suggests possibilities to couple the far-field light sources (or detectors) to symmetric modes and to optically measure symmetric modes selectively by farfield instrumentation.

\section{CONFLICT OF INTEREST}

The authors declare no conflict of interest.

\section{ACKNOWLEDGEMENTS}

We thank K Imura, K Kajikawa, K Takayanagi, Y Tanishiro and T Okamoto for valuable advice and discussion. The EELS measurement was performed with the support of S Hata at Kyushu University. This work is financially supported by Kazato Research Foundation, Kurata Memorial Hitachi Science and Technology Foundation, JSPS Kakenhi No. 26870184, and JST CREST No. 114209.

1. Maier SA. Plasmonics: the benefits of darkness. Nat Mater 2009; 8: 699-700.

2 Hess O, Pendry JB, Maier SA, Oulton RF, Hamm JM et al. Active nanoplasmonic metamaterials. Nat Mater 2012; 11: 573-584.

3 Tame MS, McEnery KR, Özdemir ŞK, Lee J, Maier SA et al. Quantum plasmonics. Nat Phys 2013; 9: 329-340.

4 Kauranen M, Zayats AV. Nonlinear plasmonics. Nat Photon 2012; 6: 737-748.

5 Nordlander P, Oubre C, Prodan E, Li K, Stockman MI. Plasmon hybridization in nanoparticle dimers. Nano Lett 2004; 4: 899-903.

6 Koh AL, Bao K, Khan I, Smith WE, Kothleitner G et al. Electron energy-loss spectroscopy (EELS) of surface plasmons in single silver nanoparticles and dimers: influence of beam damage and mapping of dark modes. ACS Nano 2009; 3 3015-3022.

7 Genet C, Ebbesen TW. Light in tiny holes. Nature 2007; 445: 39-46.

8 Alaverdyan Y, Sepúlveda B, Eurenius L, Olsson E, Käll M. Optical antennas based on coupled nanoholes in thin metal films. Nat Phys 2007; 3: 884-889.

9 Ögüt B, Talebi N, Vogelgesang R, Sigle W, van Aken PA. Toroidal plasmonic eigenmodes in oligomer nanocavities for the visible. Nano Lett 2012; 12: 5239-5244.

10 Schwind M, Zhdanov VP, Zorić I, Kasemo B. LSPR study of the kinetics of the liquidsolid phase transition in Sn nanoparticles. Nano Lett 2010; 10: 931-936.

11 Barik A, Otto LM, Yoo D, Jose J, Johnson TW et al. Dielectrophoresisenhanced plasmonic sensing with gold nanohole arrays. Nano Lett 2014; 14: 2006-2012.
12 Sigle W, Nelayah J, Koch CT, van Aken PA. Electron energy losses in Ag nanoholesfrom localized surface plasmon resonances to rings of fire. Opt Lett 2009; 34: 2150-2152.

13 Coenen T, Polman A. Optical properties of single plasmonic holes probed with local electron beam excitation. ACS Nano 2014; 8: 7350-7358.

14 Yamamoto N, Araya K, García de Abajo FJ. Photon emission from silver particles induced by a high-energy electron beam. Phys Rev B 2001; 64: 205419.

15 Yamamoto N, Ohtani S, García de Abajo FJ. Gap and mie plasmons in individual silver nanospheres near a silver surface. Nano Lett 2011; 11: 91-95.

16 Losquin A, Zagonel LF, Myroshnychenko V, Rodríguez-González B, Tencé M et al. Unveiling nanometer scale extinction and scattering phenomena through combined electron energy loss spectroscopy and cathodoluminescence measurements. Nano Lett 2015; 15: 1229-1237.

17 Adamo G, MacDonald KF, Fu YH, Wang C-M, Tsai DP et al. Light well: a tunable freeelectron light source on a chip. Phys Rev Lett 2009; 103: 113901.

18 García de Abajo FJ, Pattantyus-Abraham AG, Zabala N, Rivacoba A, Wolf MO et al. Cherenkov effect as a probe of photonic nanostructures. Phys Rev Lett 2003; 91: 143902.

19 García de Abajo FJ, Kociak M. Probing the photonic local density of states with electron energy loss spectroscopy. Phys Rev Lett 2008; 100: 106804.

20 García de Abajo FJ. Optical excitations in electron microscopy. Rev Mod Phys 2010; 82: 209-275.

21 Losquin A, Kociak M. Link between cathodoluminescence and electron energy loss spectroscopy and the radiative and full electromagnetic local density of states. ACS Photon 2015; 2: 1619-1627.

22 Knight MW, Liu LF, Wang YM, Brown L, Mukherjee S et al. Aluminum plasmonic nanoantennas. Nano Lett 2012; 12: 6000-6004

23 Coenen T, Polman A. Polarization-sensitive cathodoluminescence fourier microscopy. Opt Express 2012; 20: 18679-18691.

24 Junesch J, Sannomiya T. Ultrathin suspended nanopores with surface plasmon resonance fabricated by combined colloidal lithography and film transfer. ACS Appl Mater Interfaces 2014; 6: 6322-6331.

25 Ikenoya Y, Susa M, Shi J, Nakamura Y, Dahlin AB et al. Optical resonances in short-range ordered nanoholes in ultrathin aluminum/aluminum nitride multilayers. J Phys Chem C 2013; 117: 6373-6382.

26 Harumoto T, Sannomiya T, Matsukawa Y, Muraishi S, Shi J et al. Controlled polarity of sputter-deposited aluminum nitride on metals observed by aberration corrected scanning transmission electron microscopy. J Appl Phys 2013; 113: 084306.

27 Pettit RB, Silcox J, Vincent R. Measurement of surface-plasmon dispersion in oxidized aluminum films. Phys Rev B 1975; 11: 3116-3123.

28 Palik ED. Handbook of Optical Constants of Solids. New York: Academic Press; 1998.

29 Bigelow NW, Vaschillo A, Camden JP, Masiello DJ. Signatures of fano interferences in the electron energy loss spectroscopy and cathodoluminescence of symmetry-broken nanorod dimers. ACS Nano 2013; 7: 4511-4519.

30 Park TH, Mirin N, Lassiter JB, Nehl CL, Halas NJ et al. Optical properties of a nanosized hole in a thin metallic film. ACS Nano 2008; 2: 25-32.

31 Schmidt FP, Ditlbacher H, Hohenester U, Hohenau A, Hofer F et al. Dark plasmonic breathing modes in silver nanodisks. Nano Lett 2012; 12: 5780-5783.

32 Dahlin AB, Sannomiya T, Zahn R, Sotiriou GA, Vörös J. Electrochemical crystallization of plasmonic nanostructures. Nano Lett 2011; 11: 1337-1343.

33 Park TH, Nordlander P. On the nature of the bonding and antibonding metallic film and nanoshell plasmons. Chem Phys Lett 2009; 472: 228-231. 
34 Hayashi S, Okamoto T. Plasmonics: visit the past to know the future. J Phys Appl Phys 2012; 45: 433001.

35 Prikulis J, Hanarp P, Olofsson L, Sutherland D, Käll M. Optical spectroscopy of nanometric holes in thin gold films. Nano Lett 2004; 4: 1003-1007.

36 Parsons J, Hendry E, Burrows CP, Auguié B, Sambles JR et al. Localized surfaceplasmon resonances in periodic nondiffracting metallic nanoparticle and nanohole arrays. Phys Rev B 2009; 79: 073412.

37 Sannomiya T, Scholder O, Jefimovs K, Hafner C, Dahlin AB. Investigation of plasmon resonances in metal films with nanohole arrays for biosensing applications. Small 2011; 7: $1653-1663$.

38 Bitzer A, Ortner A, Merbold H, Feurer T, Walther M. Terahertz near-field microscopy of complementary planar metamaterials: Babinet's principle. Opt Express 2011; 19: 2537-2545.

39 Sigle W, Nelayah J, Koch CT, Ögüt B, Gu L et al. EFTEM study of surface plasmon resonances in silver nanoholes. Ultramicroscopy 2010; 110: 1094-1100.
40 Suzuki T, Yamamoto N. Cathodoluminescent spectroscopic imaging of surface plasmon polaritons in a 1-dimensional plasmonic crystal. Opt Express 2009; 17: 23664-23671.

41 Punj D, Mivelle M, Moparthi SB, van Zanten TS, Rigneault $\mathrm{H}$ et al. A plasmonic 'antenna-in-box' platform for enhanced single-molecule analysis at micromolar concentrations. Nat Nanotechnol 2013; 8: 512-516.

42 Zhang S, Genov DA, Wang Y, Liu M, Zhang X. Plasmon-induced transparency in metamaterials. Phys Rev Lett 2008; 101: 047401.

(c) (i) $\odot$ This work is licensed under a Creative Commons AttributionNonCommercial-NoDerivs 4.0 International License. The images or BY NC ND NonCommercial-NoDerivs 4.0 International License. The images or
other third party material in this article are included in the article's Creative Commons license, unless indicated otherwise in the credit line; if the material is not included under the Creative Commons license, users will need to obtain permission from the license holder to reproduce the material. To view a copy of this license, visit http:// creativecommons.org/licenses/by-nc-nd/4.0/

Supplementary Information for this article can be found on the Light: Science \& Applications' website (http://www.nature.com/lsa). 